\title{
REVISIÓN DE SEIS INSCRIPCIONES EN ALTARES VOTIVOS DEDICADOS A DEIDADES LUSITANAS Y VETONAS
}

\section{A REVIEW OF SIX INSCRIPTIONS ON VOTIVE ALTARS DEDICATED TO LUSITANIAN AND VETONIAN DEITIES}

\author{
Juan Carlos Olivares Pedreño* \\ Universidad de Alicante
}

\begin{abstract}
RESUMEN. En el presente trabajo estudiamos seis inscripciones votivas ya publicadas, procedentes de Freixo de Numáo (Guarda), Zamarra (Salamanca) y de Zarza de Granadilla, Zorita, Villamiel y Baños de Montemayor (Cáceres), pero cuyas interpretaciones actuales necesitan, a nuestro juicio, variaciones sustanciales en el nombre de los dedicantes o en los teónimos y apelativos de las divinidades citadas. Las nuevas propuestas interpretativas que desarrollamos parten de un análisis detallado realizado sobre el terreno.
\end{abstract}

PALABRAS CLAVE: Epigrafía, religión, Lusitania, vetones, altar, teónimos, onomástica.

ABSTRACT. In this paper we study six already published votive inscriptions from Freixo de Numáo (Guarda), Zamarra (Salamanca), Zarza de Granadilla, Zorita, Villamiel and Baños de Montemayor (Cáceres). In our opinion, the current interpretations about them need substantial revisions either of the names of the devotees or of the epithets and theonyms of the venerated deities. The new interpretative proposals we provide are based on a detailed analysis carried out on the ground.

KEYWORDS: Epigraphy, religion, Lusitania, altar, theonyms, Vetonians, onomastics.

* Correspondencia a / Corresponding author: Juan Carlos Olivares Pedreño, Departamento de Prehistoria, Arqueología, Historia Antigua, Filología Griega y Filología Latina. Universidad de Alicante. Campus de San Vicente del Raspeig. Ap. Correos 99 (03080 Alicante) - jc.olivares@ua.es https://orcid.org/0000-0002-8146-4901.

Cómo citar / How to cite: Olivares Pedreño, Juan Carlos (2019), "Revisión de seis inscripciones en altares votivos dedicados a deidades lusitanas y vetonas", Veleia, 36, 149-162. (https://doi.org/10.1387/veleia.19066).

Recibido: 24 enero, 2018; aceptado: 24 mayo, 2018.

ISSN 0213-2095 - eISSN 2444-3565 / (c) 2019 UPV/EHU

(c) (i) Esta obra está bajo una licencia

Creative Commons Atribución 4.0 Internacional 
El presente trabajo es el resultado de varios viajes de investigación epigráfica que llevamos a cabo durante los años 2016 y 2017, destinados a revisar varias inscripciones votivas cuyas propuestas interpretativas ofrecían, en nuestra opinión, algunas dudas importantes ${ }^{1}$. Efectivamente, el análisis sobre el terreno de dichas inscripciones nos permitió constatar que algunas de las hipótesis planteadas hasta el momento debían ser matizadas. Como es bien sabido, los altares votivos de la región occidental de Hispania ofrecen grandes dificultades de lectura por ser de granito, que es un material que se ve afectado por fuertes desgastes cuando está sometido a los agentes meteorológicos. Estos daños dificultan las lecturas en mayor medida que en las piezas de piedra caliza o arenisca, puesto que llegan a producirse frecuentes fragmentaciones en el campo epigráfico que crean gran confusión a los investigadores. Esta confusión aumenta cuando el material presenta diversos matices de color. Ello también provoca problemas añadidos ya que, en ocasiones, aunque sobre el terreno se tenga una clara convicción sobre la lectura de la inscripción, la hipótesis planteada no puede ser demostrada con claridad en la fotografía que se publica.

Todos estos problemas derivados del material a estudiar se ven incrementados cuando lo que estudiamos son altares votivos que citan a deidades paleo-hispánicas. Algunos teónimos y la mayoría de los epítetos divinos aparecen una sola vez, por lo que no se dispone de paralelos que aseguren la propuesta de lectura y muchos de estos nombres tienen una gran complejidad fonética, lo que multiplica las posibilidades interpretativas. Es por todas estas razones que hacemos nuevas propuestas de lectura para algunas inscripciones votivas que ya habían sido estudiadas por otros autores pero que, sin embargo, necesitan una nueva interpretación. Por supuesto, no es nuestra intención ofrecer estas nuevas propuestas a partir de meras alternativas posibles, sino porque creemos que los cambios, derivados de un detenido análisis, son del todo fiables.

\section{Fragmento de ara votiva procedente de Freixo de Numão (Vila Nova de Foz-Côa, GUARDA)}

Se trata del fragmento superior de un ara en granito, trabajada a cuatro caras. Solo se conserva la cabecera, fragmentada por sus esquinas frontales, y parte del fuste, que muestra un importante desgaste a consecuencia de la utilización de la pieza como parte de un muro, lo que afectó a las primeras líneas del texto. En la parte perdida solo debería constar la parte inferior de las letras de la fórmula votiva. Las medidas de la pieza son $(35) \times 35 \times 29$ y el fuste mide $(20,5) \times 26,5 \times 20 \mathrm{~cm}$. La altura de las letras se sitúa entre 3,5 y $4,5 \mathrm{~cm}$.

El monumento se hallaba formando parte de una de las paredes de la iglesia matriz de Freixo de Numão en 1992, cuando se procedió a sustituir el enlucido de la misma. En la actualidad, se conserva en el Museu Etnográfico y Arqueológico da Casa Grande, en Freixo de Numão, donde la estudiamos y fotografiamos el 20 de abril de 2017.

Se observan rastros de letras que no se habían advertido hasta ahora en $1.1 \mathrm{y}$, sobre todo, en 1. 2. Las letras que vemos nos llevan a un posible epíteto divino Cambru[.]breaegui. Este apelativo se relacionaría con algunos topónimos como Cambracum, citado en el Ravennate (IV, 43, 55) y ubicado, probablemente, en territorio cántabro (Holder, I, 1896, 716; Iglesias \& Muñiz 1992,

1 El presente estudio se enmarca en el proyecto de investigación «Sociedad romana y hábito epigráfico en la Hispania Citerior» (HAR2015-65168-P), otorgado por el Ministerio de Economía y Competitividad de España. Por otra parte, queremos mostrar nuestro más sincero agradecimiento a Jaime Río-Miranda Alcón y María Gabriela Iglesias Domínguez por su generosa ayuda durante el estudio de algunas de las inscripciones objeto de este trabajo. 
84). También conocemos el topónimo actual Cambrón, en Las Hurdes, así como otros hidrónimos y orónimos idénticos en Cáceres, Cuenca o Ciudad Real (Madoz, V, 1846, 341). También tendría relación con la mansio Cambroianna, ubicada en la región del Muro de Adriano, en Britannia (Rav., 107, 36). En el caso de Cambru[.]breaegui, habida cuenta del último elemento, -breaegui, debería referirse a un vicus o castellum de la región de Freixo de Numáo ${ }^{2}$. Otra posibilidad sería que la primera letra del apelativo fuera $S$, con lo que tendríamos un apelativo Sambru[.]breaegui, que se podría relacionar con un topónimo, muy poco fiable, citado como procedente de Aquae Flaviae (Chaves): Sambrucolensis (CIL II, 2482), aunque esa lectura es rechazada por Rodríguez Colmenero (1997, 294-295, n. $\left.{ }^{\circ} 371\right)$.

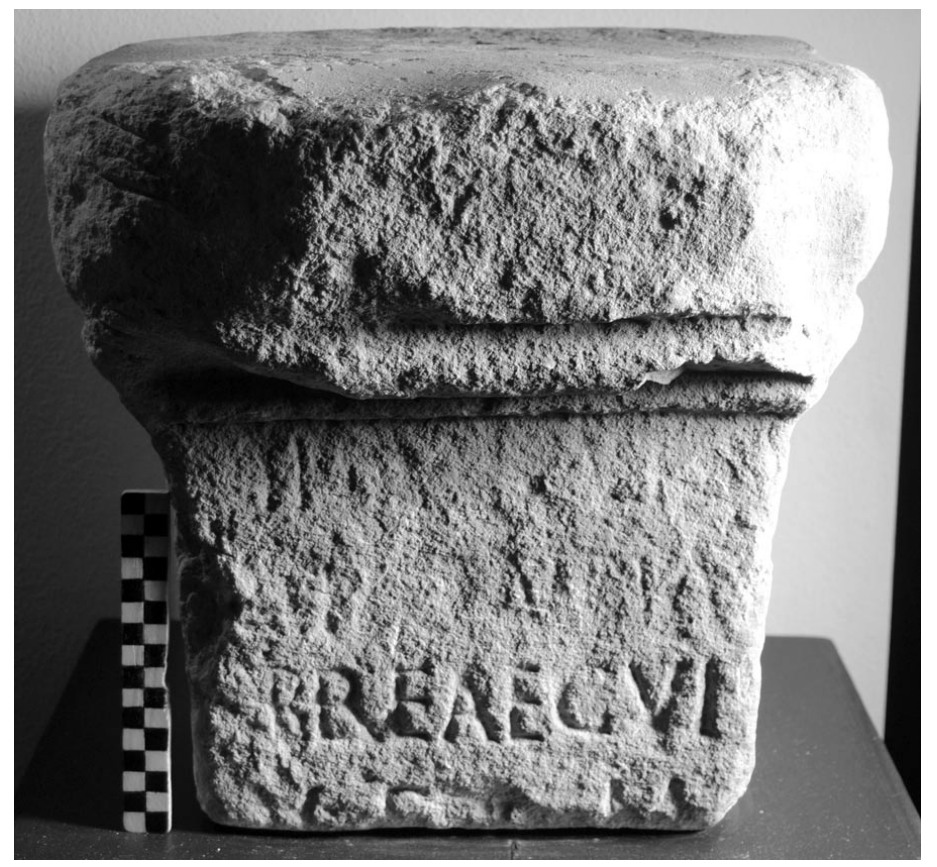
$[---] D V[---]$
[---]âmbrụu-
[.]breaegui
y(otum) ș(olvit) l(ibens) m(erito)

Figura i. Ara del Museu da Casa Grande, Freixo de Numão.

Coixão y Encarnação 1997, 4, n. ${ }^{\circ}$ 3, foto, 1. 1: [...]P(?).REAGVI (HEp 12, 648 a);

Olivares 2002, 48-49; Prósper 2002, 262.

En la primera línea, se distinguen las letras DV, que podrían llevarnos a pensar en una referencia al dios Bandue aunque, dado que tampoco es visible en el texto el nombre del dedicante y estas letras podrían formar parte del mismo, no podemos establecer una conclusión segura sobre el posible teónimo.

2 Sobre el significado de la raíz Camb-, vid. Pokorny $1959,525$. 


\section{Altar procedente de Zarza de Granadilla (Cáceres)}

Se trata de un ara en granito de color grisáceo, con base y coronamiento, trabajada a cuatro caras. En su cabecera se observa un frontón central en sus partes frontal y posterior y dos pulvinos a los lados, si bien bastante desgastados. También presenta un focus ovalado en relieve de $9,5 \times 8,5 \mathrm{~cm}$. El fuste se une a la base y a la cornisa mediante dobles boceles separados por escocias. Los laterales de la pieza fueron alineados para su reutilización, por lo que las molduras no sobresalen por los lados. Sus medidas máximas son $67,5 \times 33,5 \times 29,2$ y el fuste mide $37,5 \times 32,5 \times 25 \mathrm{~cm}$. Las letras son capitales cuadradas, aunque con algunos rasgos actuarios, con medidas entre 4 y $5 \mathrm{~cm}$.

La pieza se halló en el paramento exterior de los cimientos de la puerta de la muralla de Granadilla. Se conserva en la Casa Museo de Granadilla, donde la vimos y fotografiamos el 19 de abril de 2017.

Se han propuesto diversas alternativas sobre el teónimo y los apelativos divinos de este altar, como vemos en las referencias citadas, como la de Salas \& Burgaleta, con estructura de un teónimo y dos apelativos: Miraro Samaco Adualio. Sin embargo, era altamente sospechoso que los pretendidos epítetos fueran idénticos a antropónimos extendidos por la región lusitana. Por tanto, parece mucho más lógico interpretarlos como la referencia a la dedicante: Samacia Dualina. Estos nombres se confirman por los rastros de las letras A que aparecen al comienzo de 1.3 y l. 4. En este sentido, Samacia aparece en Villar de Plasencia, Idanha-a-Velha y Coria (Abascal 1994, 495 y Vallejo 2005, 393). Samacius aparece, además, en Silistra, en la Moesia Inferior, como nomen: Quintus Samacius Serenus (AE 1993, 942). Por otra parte, Dualina no es conocido, pero sí Dualius, que se cita en Ahigal, Cáceres (Abascal 1994, 348; Vallejo 2005, 310) y Dualia, en Conquista de la Sierra, en la misma provincia que el testimonio anterior. Samacia Dualina sería, por tanto, la madre de Rufinus, el individuo citado en la parte inferior de la ofrenda. Ella es, por tanto, quien hizo el voto, pero lo cumple su hijo.

El principal problema de esa parte final del texto es la caracterización del elemento Cada(---), que podría ser el inicio de la origo de Rufinus como, por ejemplo, Cadarnavaegiensis, a partir de un topónimo como Cadarnavaegium, que se cita en la lámina de bronce de Fuentes de Ropel, en Zamora (Mayer et al. 1998, 170). Además, se podría pensar en un nombre étnico, ya que existe un individuo que se cita como Cadaricus en la región vadiniense (Riaño, León), aunque esta posibilidad es menos probable, ya que no tenemos individuos de esta zona testimoniados en el centro de Lusitania (González Rodríguez 1986, 125, n. ${ }^{\circ}$ 65; $A E$ 1976, 303), ni étnicos que contengan ese elemento en la región.

Finalmente, hay otras opciones que conllevan aún más problemas. En este sentido, Cada(rus) es un cognomen que aparece en Crato (Portalegre) y en tres pueblos de Cáceres: Monterrubio de la Serena, Oliva de Plasencia y Valverde del Fresno (Abascal 1994, 307; Vallejo 2005, 234). Si este elemento fuera un cognomen, habría que considerar Rufinus como nomen, caso del que existe un paralelo fiable: Marcus Rufinus Albinus, citado en Mérida (Abascal 1994, 211). Otros ejemplos podrían ser Rufinus Aelatius, Rufi filius (Vaz 1990, n. ${ }^{\circ}$ 157; AE 1990, 502; HEp 4, 1103), procedente de S. Salvador (Viseu) o Rufinus Caurunius, constatado en Tres Minas, aunque el último nombre no es del todo seguro (Rodríguez Colmenero 1997, 152, n. ${ }^{\circ} 131 ; A E 1980,581$ ). Sin embargo, estos dos casos podrían ser ejemplos de una inversión del orden en el nomen y cognomen, por lo que no servirían como paralelos para considerar Rufinus como nomen. Es verdad que en la epigrafía europea tendríamos también algunos ejemplos (Schulze 1904, 221; Solin \& Salomies 1988, 15), como el de Lucius Rufinus Martialis (CIL V, 5452), de Arcisate (Italia), el de Caius Rufinus Apronius (CIL V, 5791), de Milán o el de Lucius Rufinus Phoebus (AE 1913, 201), de Albano Laziale (Italia) pero, en cualquier caso, son muy pocos casos para que podamos apostar por la opción de Rufinus como nomen y Cadarus como cognomen. 


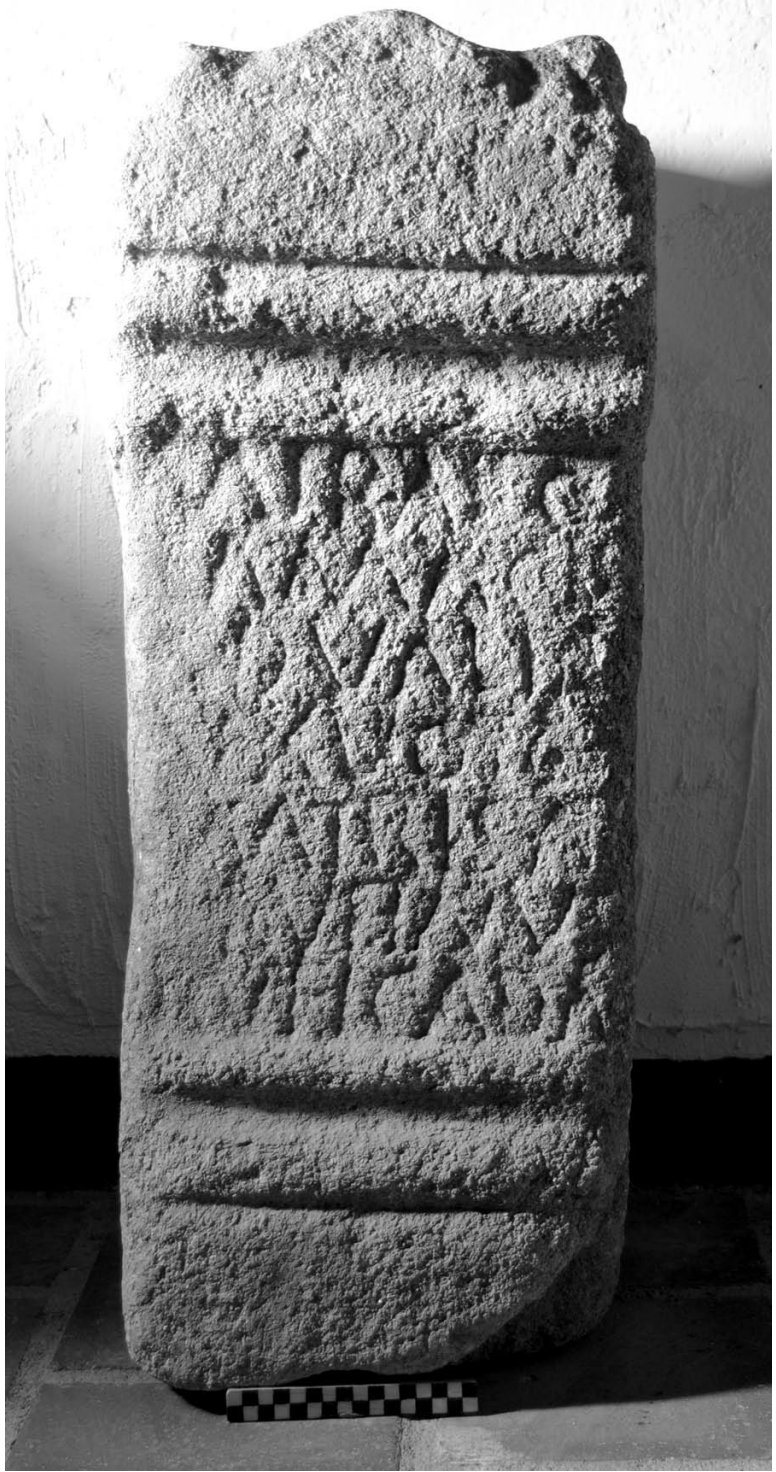

\author{
Mira[.]o \\ Samac[i]- \\ $a \cdot$ Duali[n]- \\ a ęx voto \\ [p(osuit)] Rufinus \\ Rufi (filius) Cada(---)
}

5 matris a (ram)

Figura 2. Ara de Samacia Dualina. Centro de Interpretación de Granadilla.

Salas \& Burgaleta 1996, 281-288, láms. 1-2, 1. 1-3: Miraro / Samac[o?] / Aduali[o?] (HEp 7, 263);

Olivares 2002, 35; Prósper 2002, 130; Río-Miranda 2010, 189-190, n.o 105, foto;

Esteban 2013, 187-188, n. ${ }^{\circ}$ 1139, considerándola funeraria: M(arco) Traio / Samac[i] / [f(ilio] Duali[o] / an(norum) XVo(ssa) t(ua) b(ene) / matri sua(e) / Rufinus / Rufi Cada(rus).

También se podría pensar que el individuo tenía dos cognomina: Rufinus Cadarus, ya que esta posibilidad también está constatada en la epigrafía europea, como en el caso de Lucius Iunius Rufinus Pomponianus (CIL V, 961), de Aquileia (Italia) o en el de Quintus Caecilius Rufinus Creperianus (CIL III, 10415), de Budapest (Hungría). Con todo, también son muy escasos los paralelos que permitan apoyar esta propuesta. 
En definitiva, la opción más probable sería, en nuestra opinión, considerar Cada(---) como la origo de Rufinus o, con menos probabilidad, como un étnico de este individuo. Quizá por ello está abreviado, porque el topónimo era suficientemente conocido en la región. Teniendo en cuenta todos estos datos, la última letra de 1.5 sería la abreviatura de a $(\mathrm{ram})$ y la primera de 1.6 sería la de $p$ (osuit), aunque esta última está prácticamente desaparecida. Esta solución otorga plena coherencia a la existencia del nombre del hijo, como ejecutor de la erección del altar.

En cuanto al teónimo, no podemos optar con plena fiabilidad entre Miraro y Mirato, que son las alternativas más probables, dado que en la penúltima letra del mismo hay un fuerte desgaste que impide una opción segura entre la $\mathrm{R}$ y la $\mathrm{T}$ y, además, no existen paralelos de ninguno de esos dos nombres.

\section{Altar procedente de Zorita (CÁceres)}

Es un ara en granito con base y coronamiento, trabajada a cuatro caras. La cabecera está delimitada del fuste por un bocel, mientras que el zócalo lo está mediante un rebaje. La cabecera muestra un desgaste en su parte superior derecha, aunque se ven rastros de pulvini. Sus medidas máximas son $(70) \times 27 \times 22 \mathrm{~cm}$. El fuste mide $33 \times 22 \times 19$,5. Sus letras son capitales cuadradas con rasgos rústicos y miden $3,2 \mathrm{~cm}$. Por debajo de 1.5 hay un espacio de $9,5 \mathrm{~cm}$ sin texto que se extiende hasta el comienzo del zócalo.

La pieza apareció en la dehesa boyal, a 4 kilómetros al sur de Zorita y está depositada en el Museo Arqueológico Provincial de Cáceres, donde la pudimos ver y fotografiar el 18 de abril de 2017.

El nombre de la deidad es [.]aenidivi, puesto que la interpretación de Esteban y Salas requiere algunas complicaciones epigráficas y lingüísticas difíciles de sostener, además de que, antes de la primera A de 1. 1, existe un espacio roto donde cabe una letra para ser acorde con el resto de la paginación, por lo que el teónimo no debe ser Aenidivi, como proponía Callejo, ni podría referirse a la deidad Aervina $^{3}$. Esta deidad lusitana está citada sobre un ara procedente de Salvatierra de Santiago (Fernández-Oxea 1951, 174-175, n.o 7) y sobre dos altares de Segura, en Idanha-a-Nova (Curado 1988, 6). Además, esta última interpretación, propuesta por Esteban y Salas, supondría un teónimo con un nexo RV, que es bastante insólito en la epigrafía hispana y, por otra parte, la $\mathrm{N}$ nos parece muy clara. En segundo lugar, el nombre de la deidad estaría abreviado como Aervi(nae), al igual que el supuesto apelativo Divi(nae), lo cual tampoco es habitual. Por otra parte, estas dos abreviaturas estarían unidas sin interpunción y, finalmente, el teónimo y epíteto estarían abreviados en una inscripción en la que la fórmula votiva no se abrevia, ya que los elementos votum solvit constan completos, sin que exista problema de espacio en el campo epigráfico, como ya explicamos arriba, con un espacio vacío amplio por debajo de la última línea. De hecho, si el nombre de la deidad no fuera [.]aenidivi, el lapicida habría creado una insólita confusión, al no completar los elementos ni poner interpunción entre ellos en un ámbito donde la desinencia de dativo en - ui es conocida, con testimonios procedentes de los distritos de Guarda, en Orjais (Covilhã), Queiriz (Fornos de Algodres), Freixo de Numão (Vila Nova de Foz-Côa) o Meda; de Castelo Branco, en Bemposta (Penamacor), Aldeia de Santa Margarida y Medelim (Idanha-a-Nova); de Viseu, en Freixiosa (Mangualde) y Lamas de Moledo (Castro Daire) y, finalmente, de Portalegre, en Arronches. Por último, también sería un problema aceptar el betacismo ErbinelAervinae en un territorio donde tampoco es un hecho frecuente ${ }^{4}$.

3 Queremos mostrar nuestra profunda gratitud a D. José Miguel González Bornay, por su gran disposición y amabilidad para que pudiéramos estudiar en las mejores condiciones las inscripciones del Museo Arqueológico de Cáceres.

4 Prósper 1997, 147. 


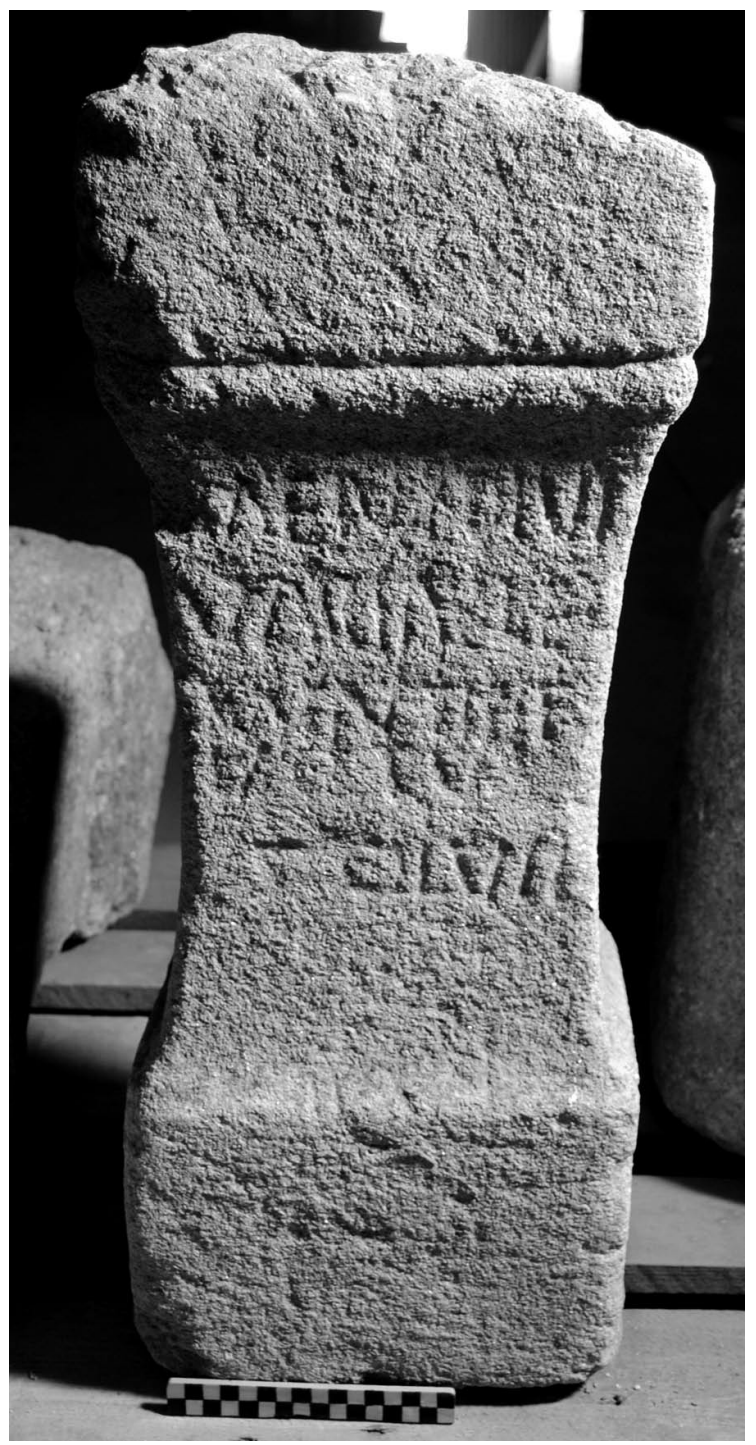

\section{[.]aenidivi Varia Cle- mentis $\cdot f($ ilia ) votu[m] solvit}

Figura 3. Ara votiva de Varia. Museo Arqueológico de Cáceres.

Callejo 1970, 155-156, n. ${ }^{\circ}$ 17, fig. 16, 1. 1-3: AENIDIVI /VARIA · [C]LE/[ME] (AE 1971, 148);

Hurtado 1977, 325, n. ${ }^{\circ}$ 766; M. Beltrán 1982, 115; Esteban \& Salas 1996-2003, 231-233, foto, lám. 2, 1. 1: Aervi(nae) Divi(nae) (AE 2002, 688; HEp 10, 145); Olivares 2002, 38; Prósper 2002, 215;

Esteban \& Salas 2003, 113-114, n. ${ }^{\circ} 120$; Esteban 2012, 317-318, n.o 897, foto y p. 508, n.o 897.

\section{Altar hallado en Villamiel (Cáceres)}

Ara en granito con base y coronamiento, trabajada a cuatro caras. La corona ha sido repicada, eliminando toda decoración de su cara superior y desgastando las molduras. Sus dimensiones son $(56) \times 26 \times 25 \mathrm{~cm}$ y el campo epigráfico mide $30 \times 20$. Las letras son capitales cuadradas, con medidas uniformes de $3,5 \mathrm{~cm}$. Las interpunciones son redondas. Fue hallada en la finca del 
Sr. Berjano, de Nava del Rey, donde también aparecieron otras inscripciones. Se conserva en el Museo Arqueológico Provincial de Cáceres, donde la estudiamos y fotografiamos el 18 de abril de 2017.

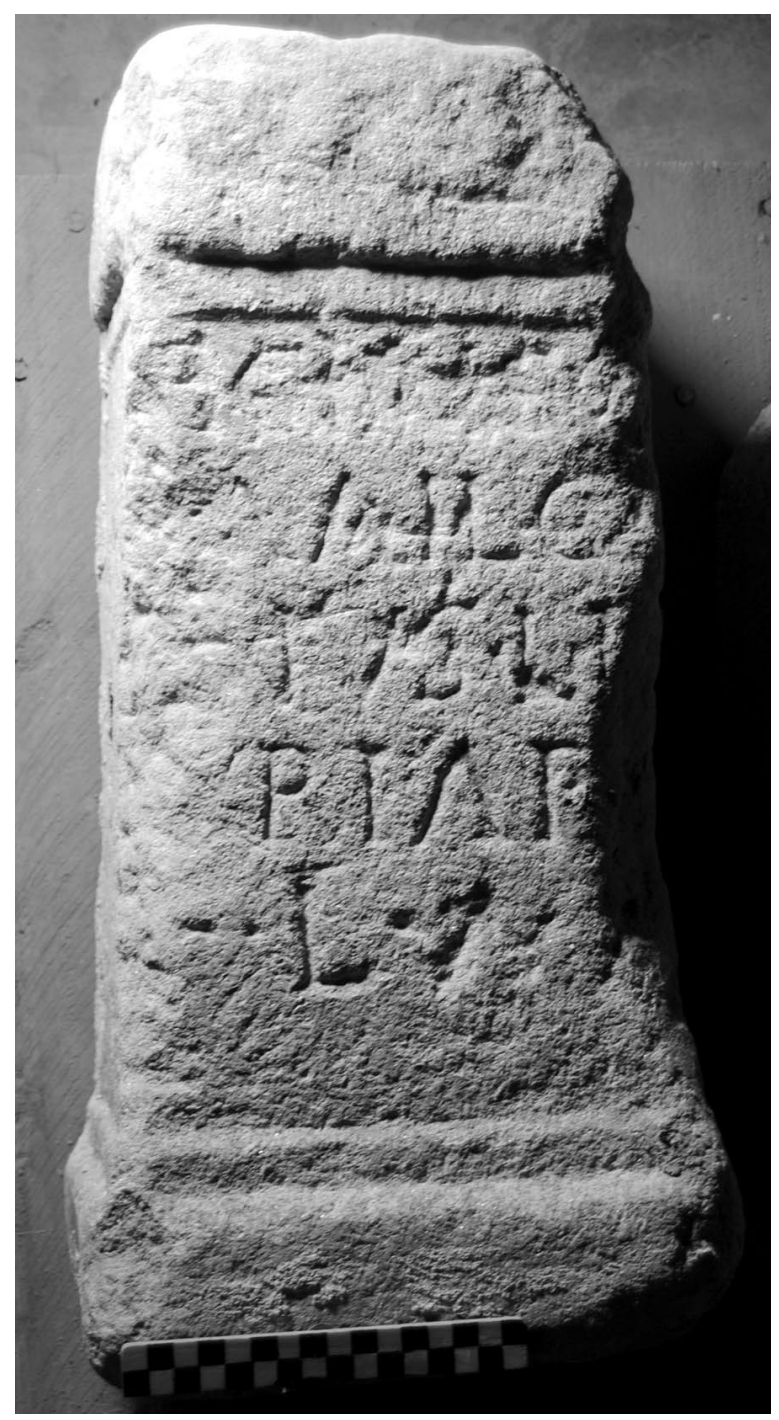

\author{
Eci[a]iso \\ Mailo- \\ nuus $\cdot$ Lẹ- \\ 5 uri (filius) a(ram) $\cdot$ p(osuit) . \\ [v(otum)] $\cdot 1$ (ibens) $\cdot$ a(nimo) $\cdot[s($ olvit $)]$
}

Figura 4. Ara de Mailonus. Museo Arqueológico de Cáceres.

Berjano 1899, 121-122, dibujo, sin lectura: [E]GN[---]V /[---]AN C / NV LI / VRIA / [E]LA;

Monsalud 1900, 322-323, n. ${ }^{\circ}$ 1: A(ppius) Claud(ius) M(arci) f(ilius), 1. 3-4: nus L(aribus) T/uri(brigensibus) a(nimo) p(osuit) / l(ibens) m(erito); Hübner, EE 9, 125, 1. 1: IGI[---]; Mallón \& Marín 1951, 90-91, n. ${ }^{\circ}$ 185, 1. 1:

EC[....], 1. 3-5: NTVS LI / RTAT / L M ; Callejo 1965, 74-78, n. ${ }^{\circ} 39$, dibujo y foto, 1. 1: EC[...] (HAE 2450);

Vives, ILER 680; Blázquez 1972, 83, n. ${ }^{\circ} 8$, 1. 1: Eg... su; Hurtado 1977, 266, n.o 639 y n.o 641;

Melena 1985, 489, n. ${ }^{\circ}$ 7, 1. 1: ECVSSV (HEp 1, 206); Piernavieja 1988, 373-374, n. ${ }^{\circ} 4$;

Madruga \& Salas 1995, 338-341, n. ${ }^{\circ}$ 6, 1. 1: ECI[r?]ICV (HEp 6, 251); García de Figuerola 1999, 158-159, n.o 4;

Olivares 2002, 39; Prósper 2002, 335; Esteban \& Salas 2003, 111-112, n. ${ }^{o} 118$, foto, 1. 1: Eci[r?]ic[u]. 
El presente texto ha dado lugar a numerosas interpretaciones, dado el fuerte desgaste de la primera línea. En nuestro estudio de la pieza, pudimos comprobar que las últimas dos letras del nombre de la deidad eran SO, lo que da mayor coherencia a la interpretación del teónimo, en dativo. El principal problema interpretativo de esta pieza consistía en que, al final de esa primera línea, hay algunos daños en la piedra que hacen vislumbrar, sobre todo si la luz viene de la izquierda, una posible V. Esto llevó a algunos autores a considerar lecturas como Ecussu o Eci[r] icu (vid. supra). Sin embargo, hemos llegado a la conclusión de que esos trazos se derivan de golpes sufridos en esa zona, dado que son muy irregulares y todas las letras seguras del monumento están ejecutadas con maestría. En primer lugar, si aceptamos esa V final, sería de la mitad de tamaño que las restantes y, además, su trazo derecho sería muy curvado e irregular. Este trazo es semejante al que se observa en el interior de la letra $\mathrm{O}$ de la segunda línea, y está causado por golpes de cincel realizados al fragmentar el lado derecho de la inscripción. A su vez, el golpe que parece un trazo izquierdo de la $\mathrm{V}$ oculta la curvatura inferior izquierda de la $\mathrm{O}$, impidiendo esa lectura. En cambio, las letras $\mathrm{S}$ y $\mathrm{O}$ son muy regulares y de perfecta ejecución, como se puede observar en la foto, aunque el desgaste de todo el campo epigráfico y las fracturas citadas impidan que se vean con total claridad. El teónimo o apelativo divino, si no hubiera letras en la cabecera, algo que no se puede rechazar de plano, sería Eciaiso y carecería de paralelos hasta el momento.

\section{Altar hallado en Zamarra (Salamanca)}

Ara en arenisca, con zócalo y cabecera, trabajada a cuatro caras y con inscripción en dos de ellas. La cornisa es moldurada, con tres boceles. El zócalo presenta un bocel y, por encima de este, una superficie plana descendente hasta el fuste. Sus dimensiones totales son $60 \times 37 \times 25 \mathrm{~cm}$. Las letras son capitales actuarias, con rasgos rústicos y miden entre 3 y $4 \mathrm{~cm}$. Las interpunciones son circulares. La pieza procedería de Lerilla, al suroeste de Zamarra, según Hernández Guerra. Se conserva en el Museo Catedralicio de Ciudad Rodrigo.

Nuestra interpretación difiere claramente de la propuesta por Hernández Guerra. En nuestra opinión, la lectura que presentamos es muy probable a partir de nuestro estudio sobre el terreno. Aiburina no está testimoniado hasta el momento, aunque sí lo están Aeburina, Aibarus, Aebarus, Aebura, Eburusla, Ebureinius, Eburenius y Eburianus (Abascal 1994, 258 y 349; Vallejo 2005, 108-109 y 313-314). Las interpunciones que permiten delimitar en el texto su estatuto de liberta son bastante claras. En cuanto al resto de la inscripción, seguimos la lectura de Hernández Guerra. 


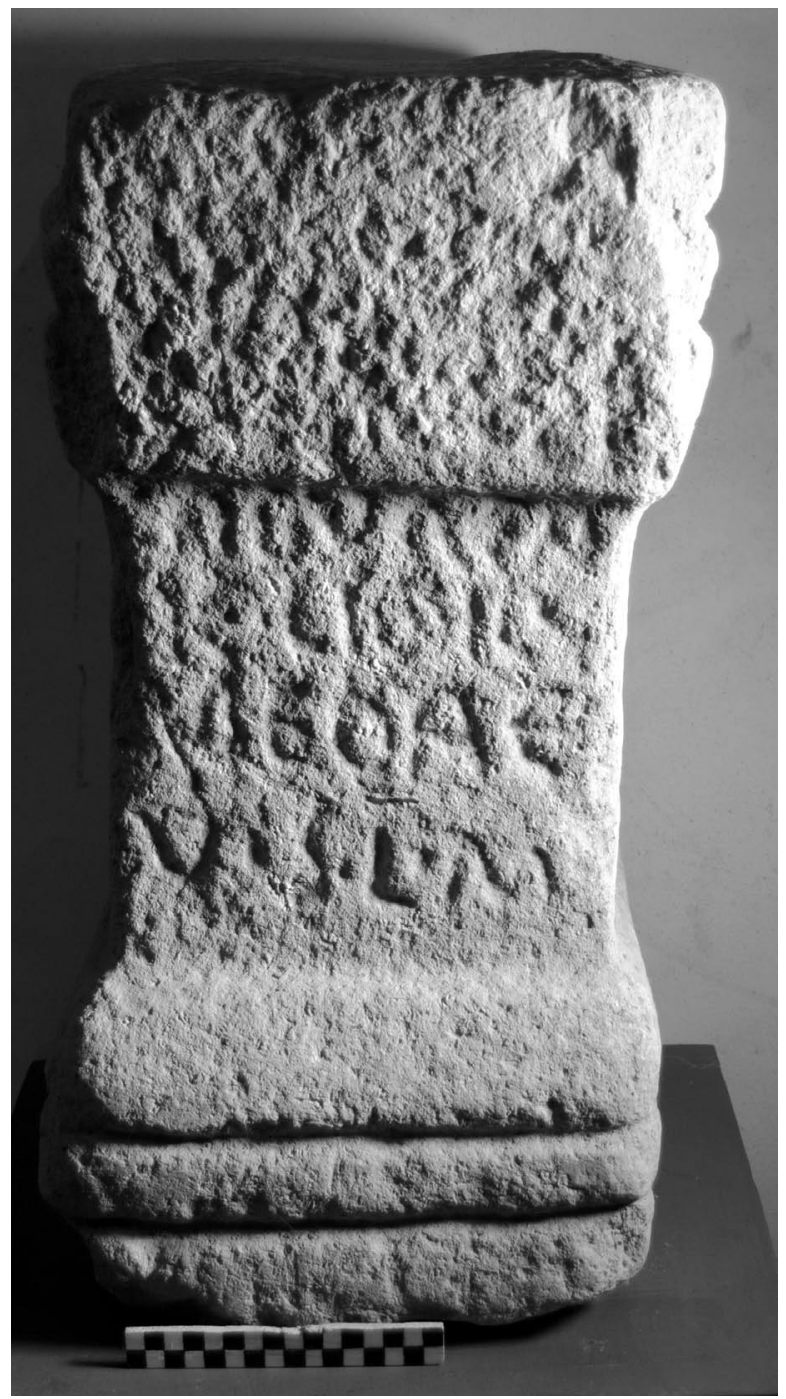

\author{
Aiburin- \\ $a \cdot C($ ai $) \cdot \operatorname{lib}($ erta $) \cdot I l u-$ \\ rbedae \\ v(otum $) \cdot s($ olvit $) \cdot 1($ ibens $) \cdot m($ erito $) / /$ \\ [Ilurbedae?] \\ Sacrum \\ v(otum) s(olvit) l(ibens) m(erito)
}

Figura 5. Ara de Aiburina. Museo Catedralicio de Ciudad Rodrigo.

Hernández Guerra 2001, 25-26, n. ${ }^{\circ}$ 14, lám. 4, 14, 1. 1-2: QVADRATVS / SV(a)E ILVR (HEp 11, 430).

\title{
6. Ara hallada en Baños de Montemayor (Cáceres)
}

Ara en granito con zócalo y con la cabecera muy desgastada. Sus medidas son $(75) \times(28)$ y el fuste mide $38,4 \times 26 \mathrm{~cm}$. Las letras son capitales cuadradas, de $4 \mathrm{~cm}$ en todas sus líneas. Las interpunciones son circulares. Se desconoce su procedencia exacta y, en la actualidad, está insertada en el muro orientado al noroeste del atrio de la iglesia parroquial de Santa María. 


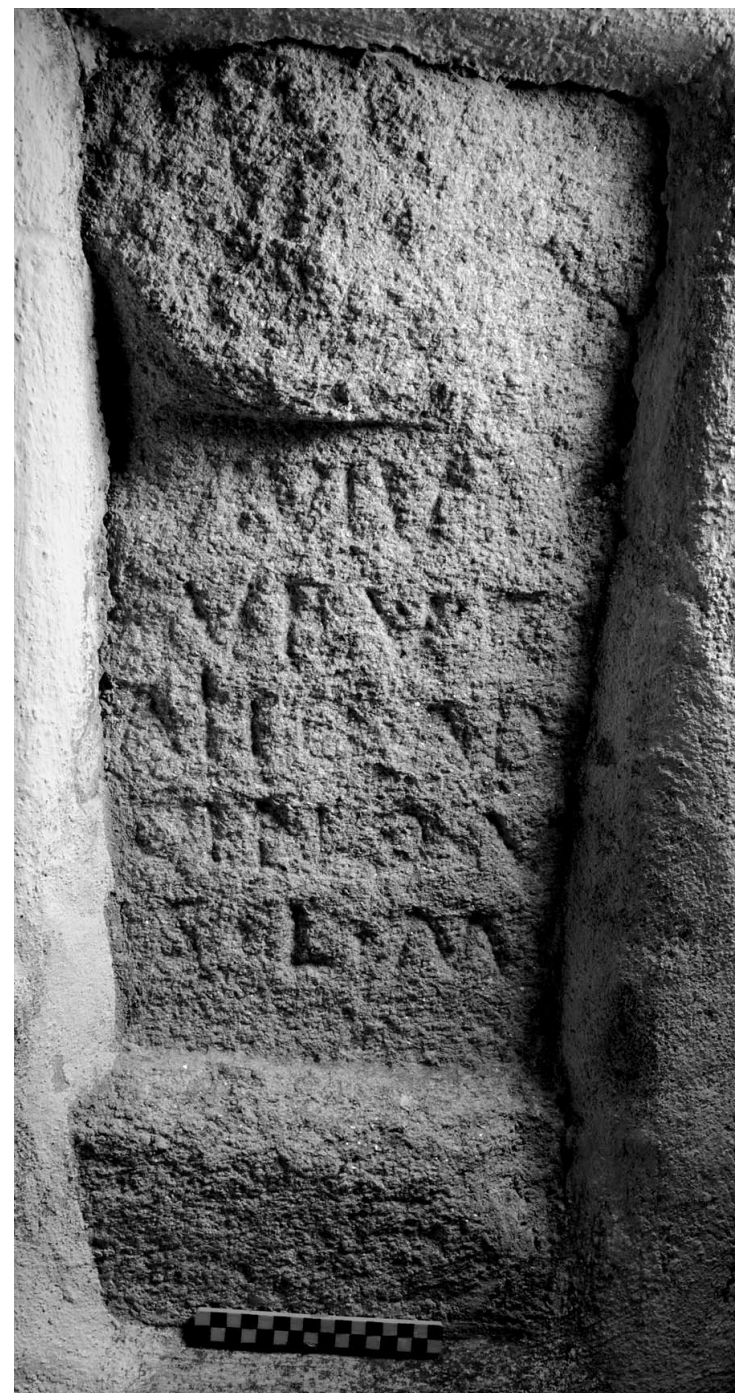

Viviu[s]

[R]ufu[s]

Aitio Ad-

cino $\cdot v($ otum)

$s$ (olvit) $\cdot 1($ ibens $) \cdot m$ (erito) .

Figura 6. Inscripción de Vivius Rufus, en la Parroquia de Santa María de Baños de Montemayor.

Sánchez Paredes, Diario Extremadura 26/7/1966, n. ${ }^{\circ}$ 7, dibujo; Blázquez 1975, 25;

Hurtado 1977, 347, n. ${ }^{\circ} 821$, quien solo registra el teónimo; Haba 1986, 89-91, n. o 40; Olivares 2002, 54;

Esteban 2013, 49-50, n. 949 , foto, 1. 1-4: [Vi]viu(s) / [R]ufu[s] / Aiioad[.?]/cino $\cdot v($ otum). 


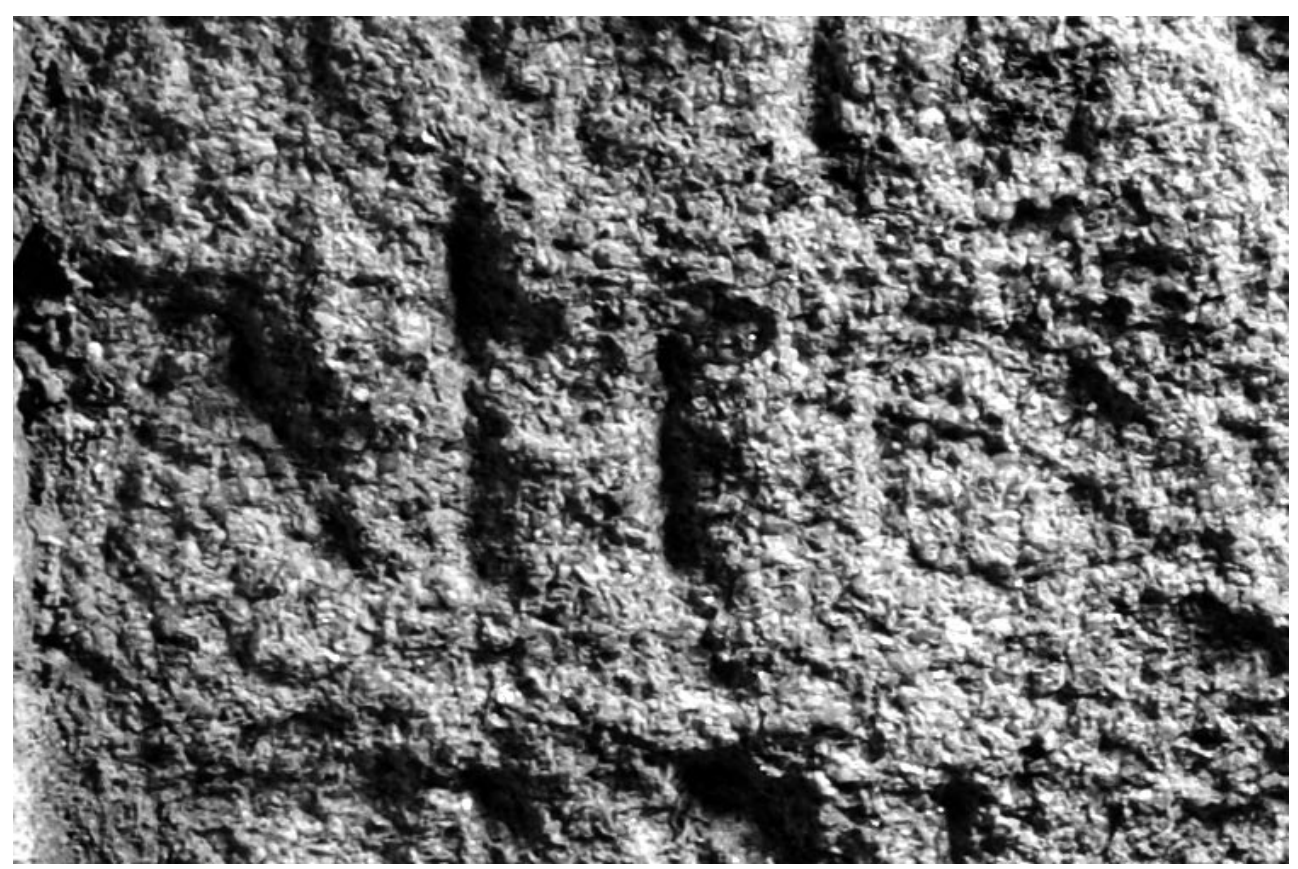

Figura 7. El teónimo Aitius, con detalle del nexo IT.

La interpretación de Esteban suponía un gran avance respecto a lecturas anteriores. Sin embargo, observamos en 1. 3 un nexo IT que supone una nueva interpretación del teónimo: Aitius, conocido en Lusitania por dos inscripciones, aunque en la forma Aetius, en la región de Fundão y Sabugal. Esta variante fonética no es extraña en la zona, dado que conocemos los antropónimos, como hemos visto arriba, Caesaro y Caisaro, Aebarus y Aibarus, Aeburina y Aiburina, pero también Andaetius y Andaitius (Vallejo 2005, 153), Caeno y Caino (Ibid., 240), Maelo y Mailo (Ibid., 341343) o Saelgius y Sailgius (Ibid., 391).

Según la interpretación de lectura de Esteban, el teónimo podría haber sido el mismo que el de las inscripciones de Hontangas (Abásolo 1973, 443-444), Clunia (Palol \& Vilella 1987, 161-162, n. ${ }^{\circ}$ 209) y Villafranca de Montes de Oca (Abásolo, 1984, 199-200, n. $\left.{ }^{\circ} 11 ; A E 1984,569\right)$, es decir, Aeio, Aeo o Aio (dat.), lo que habría tenido ciertas consecuencias. En este sentido, habría sido necesario suponer una migración o transmisión cultural desde el área celtibérica hasta la región lusitana lo suficientemente importante para que se hubiera erigido un altar en un lugar público a la deidad celtibérica. En cambio, la nueva interpretación sitúa el teónimo lusitano Aetius o Aitius en un área más extensa, pero dentro del ámbito cultural lusitano lo que, en principio, es más lógico según los datos que tenemos hasta el momento sobre la extensión de las áreas de culto de las deidades lusitanas.

El nomen latino Vivius está registrado en Cádiz y Sevilla y el cognomen Rufus es de los más extendidos de la península ibérica (Abascal 1994, 249 y 485-490). 
REVISIÓN DE SEIS INSCRIPCIONES EN ALTARES VOTIVOS DEDICADOS A DEIDADES LUSITANAS Y... 161

\section{BibliografíA}

Abascal, J. M., 1994, Los nombres personales en las inscripciones latinas de Hispania, Madrid-Murcia: Universidad Complutense de Madrid-Universidad de Murcia.

AвÁsolo, J. A., 1973, «El árula de Hontangas, la inscripción de Cuevas de Amaya y la estela de Fresneda de la Sierra (Burgos)», BSAA 39, 443-449.

AвÁsolo, J. A., 1984, «Recientes hallazgos de lápidas romanas en la provincia de Burgos», BSAA 50, 195-216.

Beltrán, M., 1982, Museo de Cáceres. Sección de Arqueología, Cáceres: Ministerio de Cultura.

Berjano, D., 1899, «Nota epigráfica», Revista de Extremadura 1 (cuad. II, marzo), 121-122.

BlázQuez, J. M., 1972, «Últimas aportaciones al estudio de las religiones primitivas de Hispania», Homenaje a Antonio Tovar, Madrid: Gredos, 81-90.

Blázquez, J. M., 1975, Diccionario de las religiones prerromanas de Hispania, Madrid: Ediciones Istmo.

Callejo, C., 1965, «Aportación a la epigrafía romana del campo norbense», BRAH 157, 11-82.

Callejo, C., 1970, «Nuevo repertorio epigráfico de la provincia de Cáceres», $A E A$ 43, 132-168.

Coixẫo, A., \& J. D’EnCarnação, 1977, Foz Côa romana: notas epigráficas (Exposição, de 13 de Setembro a 31 de Dezembro de 1997, integrada nas comemoraçóes dos 700 anos do tratado de Alcanizes, 1297-1997), Vila Nova de Foz Côa: Câmara Municipal de Vila Nova de Foz Côa.

Curado, F. P., 1988, "As aras da capela de Santa Marinha (Segura, Idanha-a-Nova)», O Raiano, año XVI, n. ${ }^{\circ} 166$, octubre, 6 .

Esteban, J., 2012, Corpus de inscripciones latinas de Cáceres. II. Turgalium, Cáceres: Universidad de Extremadura.

Esteban, J., 2013, Corpus de inscripciones latinas de Cáceres. III. Capera, Cáceres: Universidad de Extremadura.

Esteban, J., \& J. Salas, 1996-2003, «Puntualizaciones y correcciones a la epigrafía de Cáceres», Norba 16, 227-240.

Fernández OXEA, J. R., 1951, «De epigrafía cacereña», BRAH 128, 165-195.

García de Figuerola, M., 1999, Arqueología romana y altomedieval de la Sierra de Gata, Cáceres: Universidad de Extremadura.

Нава, S., 1986, Catálogo epigráfico del partido judicial de Plasencia, Plasencia.

Hernández Guerra, L., 2001, Epigrafía de época romana de la provincial de Salamanca, Valladolid: Universidad de Valladolid.

Holder, A., 1896, Alt-Celtischer Sprachschatz, vol. I, Leipzig: B.G. Teubner.

HüBner, E., 1899, Ephemeris Epigraphica; Corporis Inscriptionum latinarum supplementum, vol. IX, Berlín.

Hurtado, R., 1977, Corpus provincial de inscripciones latinas. Cáceres, Cáceres: Diputación provincial.

Iglesias, J. M., \& J. A. MuÑIz, 1992, Las comunicaciones en la Cantabria romana, Santander: Librería Estudio.

Madoz, P., 1846, Diccionario Geográfico-Estadistico-Histórico de España y sus Posesiones de Ultramar, t. V, Madrid: Est. Literario-Tipográfico de P. Madoz y L. Sagasti.

Madruga, J. V., \& J. Salas, 1995, "A propósito de teónimos indígenas en el Conventus Emeritensis», ETF serie II, 8, 331-355.

Mallón, J., \& T. Marín, 1951, Las inscripciones publicadas por el Marqués de Monsalud (1897-1908). Estudio crítico, Madrid: CSIC. Instituto Antonio de Nebrija de Filología.

Mayer, M., R. García \& J. A. Abásolo, 1998, «El bronce de Fuentes de Ropel (Zamora)», BSAA 64, 161 174.

Melena, J. L., 1985, «Salama, Jálama y la epigrafía latina del antiguo corregimiento», Symbolae Ludovico Mitxelena septuagenario oblatae, Vitoria-Gasteiz: Instituto de Ciencias de la Antigüedad, Universidad del País Vasco, 475-530.

Monsalud, M., 1900, «Nuevas inscripciones romanas de Extremadura», BRAH 37, 322-325.

Olivares, J. C., 2002, Los Dioses de la Hispania Céltica, Madrid: Real Academia de la Historia-Universidad de Alicante.

Palol, P., \& J. Vilella, 1987, «Clunia II. La Epigrafía de Clunia», EAE 150. 
Piernavieja, P., 1988, «Denudator gimanasi u.s. Arescu», Anejos Gerion 1, 359-379.

РокоRny, J., 1959, Indogermanisches Etymologisches Wörterbuch, Viena: Francke.

Prósper, B., 1997, «El nombre de la diosa lusitana Nabia y el problema del betacismo en las lenguas indígenas del Occidente peninsular", 'Ilu. Revista de Ciencias de las Religiones 2, 141-149.

Prósper, B., 2002, Lenguas y religiones prerromanas del occidente de la Península ibérica, Salamanca: Universidad de Salamanca.

Río-Miranda, J., 2010, La Ciudad Romana de Cáparra. Municipium Flavium Caparense, Pamplona.

Rodríguez Colmenero, A., 1997, Aquae Flaviae I. Fontes epigráficas da Gallaecia meridional interior (2. ${ }^{\text {a }}$ ed.), Chaves: Câmara Municipal de Chaves.

Salas, J., \& J. Burgaleta, 1996, "Miraro Samaco Adualio, una nueva divinidad vettona», ETF (serie II) 9, 281-288.

Sánchez Paredes, A., 1966, «Inscripciones latinas de la Vettonia», Diario Extremadura 26/7.

Schulze, W., 1904, Zur Geschichte lateinischer Eigennamen, Berlin: Weidmannsche Buchhandlung.

Solin, H., \& O. Salomies, 1988, Repertorium nominum gentilium et cognominum Latinorum, HildesheimZürich-New York: Olms-Weidmann.

Vallejo, J. M., 2005, Antroponimia indígena de la Lusitania romana, Vitoria: Universidad del País Vasco.

VAZ, J. L. I., 1990, "Ara votiva a Albucelaincus», FE 35, n. ${ }^{o} 157$.

VIVES, J., 1971, Inscripciones latinas de la España romana, Barcelona: CSIC-Universidad de Barcelona (ILER). 